\title{
SOX2OT knockdown derived changes in mitotic regulatory gene network of cancer cells
}

\author{
Marie Saghaeian Jazi ${ }^{1}$, Nader Mansour Samaei ${ }^{2 *}$, Seyed Javad Mowla ${ }^{3}$, Babak Arefnezhad ${ }^{4}$ \\ and Morteza Kouhsar ${ }^{5}$
}

\begin{abstract}
Background: SOX2 overlapping transcript (SOX2OT) is a long non-coding RNA, over-expressed in human tumor tissues and embryonic cells. Evidences support its function in the cell cycle; however there is no clear mechanism explaining its function in cell proliferation regulation. Here we investigated cancer cell response to SOX2OT knockdown by RNA sequencing.

Methods: SOX2OT expression was inhibited by siRNA in two cancer cell lines (A549, U-87 MG), then the RNA of treated cells were used for the cDNA library synthesis and RNA sequencing. The differentially expressed genes were used for functional enrichment and the gene expression network was analyzed to find the most relevant biological process with SOX2OT function. Furthermore, the expression change of candidate genes was measured by qRT-PCR for more confirmation and the cell cycle was monitored by PI staining.

Results: Our findings showed that SOX2OT knockdown affects the cellular gene expression generally with enriched cell proliferation and development biological process. Particularly, the cell cycle and mitotic regulatory genes expression including: CDK2, CDK2AP2, ACTR3, and chromosome structure associated genes like SMC4, INCENP and GNL3L are changed in treated cancer cells.
\end{abstract}

Conclusion: Our results propound SOX2OT association with cell cycle and mitosis regulation in cancer cells.

Keywords: SOX2 overlapping transcript, Cell cycle, Cancer cell

\section{Background}

Long non-coding RNAs (lncRNAs) are mRNA like ribonucleic acids with no protein products. Generally, they act in a wide range of cellular and molecular processes including chromatin remodeling [1-3], gene regulation $[4,5]$, proliferation $[6,7]$, metastasis $[8-10]$ and etc. As respect to their key functions; there are many lncRNAs reported to be associated with human diseases [11-13].

SOX2OT is a lncRNA located in chr3q:26which overlaps SOX2 gene in sequence $[14,15]$. The SOX2OT expression is de-regulated in human cancer tissues

\footnotetext{
*Correspondence: samaei@goums.ac.ir

2 Stem Cell Research Center, Golestan University of Medical Sciences, Po Box: 4934174611, Gorgan, Iran

Full list of author information is available at the end of the article
}

[16-18] and its expression decrease during differentiation of cells $[14,18]$. Considering the concordant expression of $S O X 2$ with its overlapping, It has been suggested that SOX2OT functions in SOX2 regulation [18]. There are also some evidences supporting its function in regulation of the cell cycle in a polycomb-group protein, EZH2 dependent manner [17]. However, the underlying mechanism of SOX2OT function in cancer progression and differentiation appeals more investigations.

Preliminarily, we investigated two transcriptome resources to find out the most appropriate sample origin for SOX2OT functional analysis. According to the GENEVESTIGATOR software [19], SOX2OT gene expression is mostly reported to be de-regulated in brain and lung tumors (Additional file 1: Figure S1A). indeed, in a computationally reconstructed portrayal of human 
transcription database resource (MiTranscriptome) [20]; $S O X 2 O T$ expression is reported to be mostly associated with the two cancer types of glioblastoma and lung carcinoma (Additional file 1: Figure S1B). Previously in our laboratory, we observed that SOX2OT inhibition can significantly decrease lung [21] and brain (un-published yet) cancer cell colony formation ability with a minor cell cycling disturbance. Then in this study, we aimed to explore the transcriptome changes in the SOX2OT knocked down glioblastoma and lung adenocarcinoma cell lines with the RNA sequencing to clear the cellular function of SOX2OT long non-coding RNA in cancer cells.

\section{Methods}

\section{Cell culture}

A549, human lung adenocarcinoma cancer cell line and U87-MG, human glioblastoma cell line were obtained from pasture institute (Tehran, Iran). Cells were cultured in RPMI 1640 (Invitrogen, Gaithersburg, MD) supplemented with $10 \%$ fetal bovine serum (Invitrogen, Gaithersburg, MD) and $100 \mathrm{IU}$ penicillin-100 $\mu$ g streptomycin per ml (Invitrogen, Gaithersburg, MD) in a 98\% humidified $5 \% \mathrm{CO}_{2}$ incubator. The cancer cells were used for transfection process with siRNA and gene expression analysis as following.

\section{RNA interference and transfection}

Considering previous recorded high expression level of SOX2OT in cancer cells, RNA interference approach was used to investigate SOX2OT associated cellular functions. A previously reportedSOX2OTtargetingsiRNA (5'-GGAGAUUGUGACCUGGCUU-3') [18] was synthesized. For the siRNA transfection, approximately $5 \times 10^{5}$ cells were seeded at six well tissue culture plates. After $24 \mathrm{~h}$, the cells (about $80 \%$ confluent) were transfected with control siRNA (Sanatacruze, s-c37007, $10 \mathrm{nM}$ ), or SOX2OT targeting siRNA (synthesized by Bioneer, $50 \mathrm{nM}$ ) using Lipofectamine 2000 according to manufacturer's protocol. $48 \mathrm{~h}$ later, the cells were harvested and were stored in $-80{ }^{\circ} \mathrm{C}$ for subsequent gene expression analysis or RNA sequencing.

\section{RNA-sequencing}

The total RNA of control and treated A459 and U87-MG cells were extracted by Trizol reagent (Invitrogen, Carlsbad, CA) according to manufacturer's protocol. The RNA libraries were prepared after rRNA cleanup, according to the BGI standard pipeline. Sequencing of the four synthesized RNA libraries were carried out by Illumina Hiseq 2000 sequencing system with paired end sequencing method resulted to approximately $25,000,000$ reads with 90 base pairs fragments in length.
Primary sequences were checked for quality and then were aligned to the reference human genome (hg38) release using the bowtie 2 [22] tool. To find out the differentially expressed genes (DEGs) between control-siRNA and SOX2OT-siRNA treated of each cell line separately, TopHat and Cufflinks pipeline was executed. The normalized abundance of the transcripts were reported as FPKM (Fragments Per Kilobase of transcript per Million mapped reads) and the differential gene expression was evaluated using Cuffdiff package (q-value $<0.05)$ [23].

\section{Functional enrichment of DEGs and network construction} The differently expressed gene lists (up-regulated or down-regulated) were used for gene ontology (GO) term enrichment by BINGO [24] software. For more confirmation, the common DEGs ( $\mathrm{n}=208$ in both cell lines) were extracted and used for gene network analysis. The common DEGs were used for network construction using STRING protein interaction database [25] (with confidence score $=0.5$ ) and then the resulted primary network (with 86 nodes) was again extended by GeneMANIA [26] according the co-localization, protein and genetic interaction, pathways and shared protein domains of the common DEGs resulting to more dense gene network (nodes $=196,171$ nodes in DEGs +25 nodes added automatically). The edges in the final gene network was weighted based on biological process domain of GO and semantic similarity measure. The semantic similarity between genes was calculated based on rensik [27] method by using FastSemSim [28] tool (mixing strategy $=\max$ ). Finally, the weighted gene network (weight $\geq 0.4$ ) with 122 nodes was analyzed and visualized with Cytoscape 3.4 software.

\section{Gene expression measurement}

To confirm the gene expression changes, seven genes enriched mainly in cell cycle regulation or mitotic progression were measured by more sensitive method such as qRT-PCR. Briefly, one microgram of total RNAs were DNaseI (Thermo Fisher Scientific, Inc) treated and were reverse transcribed using PrimeScript first strandcDNA synthesis kit (Takara) and random hexamer primers as described by the supplier. A volume of $2 \mu \mathrm{l}$ of first strand cDNAs were used as template of real-time PCR.

The gene Expression analysis was carried out in Bioer (LineGene K) thermo cycler using qPCR Green Master with low ROX (Jena Bioscience, $\mathrm{GmbH}$ ) and specific primers (Table 1). The cycling condition was as follow: enzyme activation at $95^{\circ} \mathrm{C}(2 \mathrm{~min}), 40$ cycles of denaturing at $95^{\circ} \mathrm{C}(10 \mathrm{~s})$, annealing at $60^{\circ} \mathrm{C}(30 \mathrm{~s})$ for $G N L 3 l$ and SOX2OT; for the other genes; annealing at $58.4{ }^{\circ} \mathrm{C}(30 \mathrm{~s})$, following extension at $72{ }^{\circ} \mathrm{C}(40 \mathrm{~s})$. Final dissociation 
Table 1 The primers sequence for q-PCR amplification

\begin{tabular}{ll}
\hline Gene symbol & Primer sequence \\
\hline SOX2OT & F: GGCTGGGAAGGACAGTTCG \\
Gene ID: 347689 & R: AGATGATCTTGCCAGGCGATC \\
CDK2 & F: CCCTTTCTTCCAGGATGTGA \\
Gene ID: 1017 & R: TCACCCCTGTATTCCCAGAG \\
CDK2AP2 & F: TGCCAGGCACTCTCTGACTA \\
Gene ID: 10263 & R: AGATCCGGCCTACCTATGCT \\
GNL3L & F:TATCTTCTTGTGGCCCTTGG \\
Gene ID: 54552 & R: AGAGAGCAAGCAGATTTGACC \\
ACTR3 & F:TTGAGTGGTGGTAGATTGAAGC \\
Gene ID: 10096 & R: CCAAACTGCATATCGCTGCAT \\
SMC4 & F:TGCAGAGGAATCCTTACCAG \\
Gene ID: 10051 & R:TGTTCAGCAATGTGACCATC \\
INCENP $: 3619$ & F:TCAGAACCAACTTTCTGGGG \\
Gene ID: 3619 & R: CAAGAAGACTGCCGAAGAGC \\
GAPDH & F: GAGCGAGATCCCTCCAAAAT \\
Gene ID: 2597 & R: GGCTGTTGCATACTTCTCATG \\
\hline
\end{tabular}

curve analysis was performed to ensure the specific PCR products.

\section{Flowcytometry analysis}

To investigate the cellular apoptosis and cell cycle progression, cells were harvested and then were stained with the annexin V/PI (Sigma-Aldrich) according the manufacturer's recommendation for apoptosis evaluation; or were fixed in cold ethanol (70\%) and then were stained with stain solution (PI $10 \mu \mathrm{g} / \mathrm{ml}$ ) after treatment with triton $\mathrm{X}-100(0.1 \%)$ and RNAaseI $\left(10 \mu \mathrm{l} / \mathrm{ml}, 30 \mathrm{~min}, 37^{\circ} \mathrm{C}\right)$ for cell cycle assessment with the flowcytometry instrument (Partec GmbH, Münster, Germany).

\section{Statistics}

The gene expression measurements were carried out in experimental replicates to decrease the artificial error. The SPSS v22 software was used to analyze data statistically. For statistical tests, 95\% confidence interval and $\mathrm{p}$ value $<0.05$ was considered. The one way ANOVAWAS used to compare means of measurements in treated cells to control. The mean $\pm \mathrm{SE}$ has been presented in the graphs as error bars. For functional enrichment, the $\mathrm{p}$ value was corrected via false discovery rate (FDR) estimation.

\section{Results}

\section{SOX2OT knock down leads to general gene expression} de-regulation in both cancer cells

The RNA-sequences were analyzed as described above to investigate the differentially expressed genes for each cell line separately. More than 50,000 genes were mapped in transcriptome analysis, with 1588 genes significantly deregulated in A549 (779 up and 809 down) and 609 genes significantly de-regulated in U87-MG (391 up and 218 down) after SOX2OT knockdown. As it is visualized by histogram and scatter plots in Fig. 1, SOX2OT knockdown generally changes the expression profile of each cancer cell affecting A549 lung cancer cell more than glioblastoma U87-MG cancer cell. However, regarding to the volcano plot, the fold change expression of some of DEGs is considered as significant.

The GO term enrichment of the significantly de-regulated genes in each cell line (Table 2) indicted that most of the enriched gene ontology terms are related to the cell cycle, DNA replication, and mitosis in A549 cell line (bolded in Table 2). More ever, in the U87-MG cell line, SOX2OT knockdown changes the expression of cellular genes related to neuronal differentiation, development and also cell cycle regulation and DNA replication (bolded in Table 2). These findings highlight the potential function of SOX2OT in cellular replication and mitosis process and cellular differentiation and development. Since the two sequenced cell lines are from different tissue origin with consequently different background transcriptome, it may describe the observed variances of DEGs between two samples.

To investigate the DEGs function, the top 400 genes of the expression signature profile of DEGs in U-87 MG cell line with $\mathrm{p}$-value $\leq 0.02$ was used as a template signature to find out the most similar signatures available in the public data bases using the GENEVESTIGATOR software (by Euclidean distance score). Interestingly we found that the log2 expression of the DEGs in U87-MG cell line after SOX2OT knock down is mostly similar to studies with Asthma perturbation (relative similarity score $=1.6$ ), hypoxia (relative similarity score $=1.4$ ) and exposure to cell cycle inhibitor chemicals like mitomycin and colchicine (relative similarity score $>1.3$ ) (Additional file 1: Figure S2).

Furthermore, DEGs signature in the A549 cell after SOX2OT knock down mostly resembles conditions like: human ovarian tumors (relative similarity score $=1.28$ ), smoking (relative similarity $=1$ ), asthma (relative similarity $>1$ ), hypoxia (relative similarity score $=1.15$ ) and exposure to the anti-cancer drugs like: R549, (CDK inhibitor), echinomycin (HIF inhibitor) and zalypsis (double strand break inducer) (relative similarity score $>1.1$ ) (Additional file 1: Figure S3). Studying the most resembling perturbation signatures can provide more clues to find out the importance of SOX2OT gene function in human diseases.

\section{Common genes de-regulated in both SOX2OT knocked down cancer cell lines}

For more precision, we searched the differentially expressed genes in each cell line to find out the sharing 


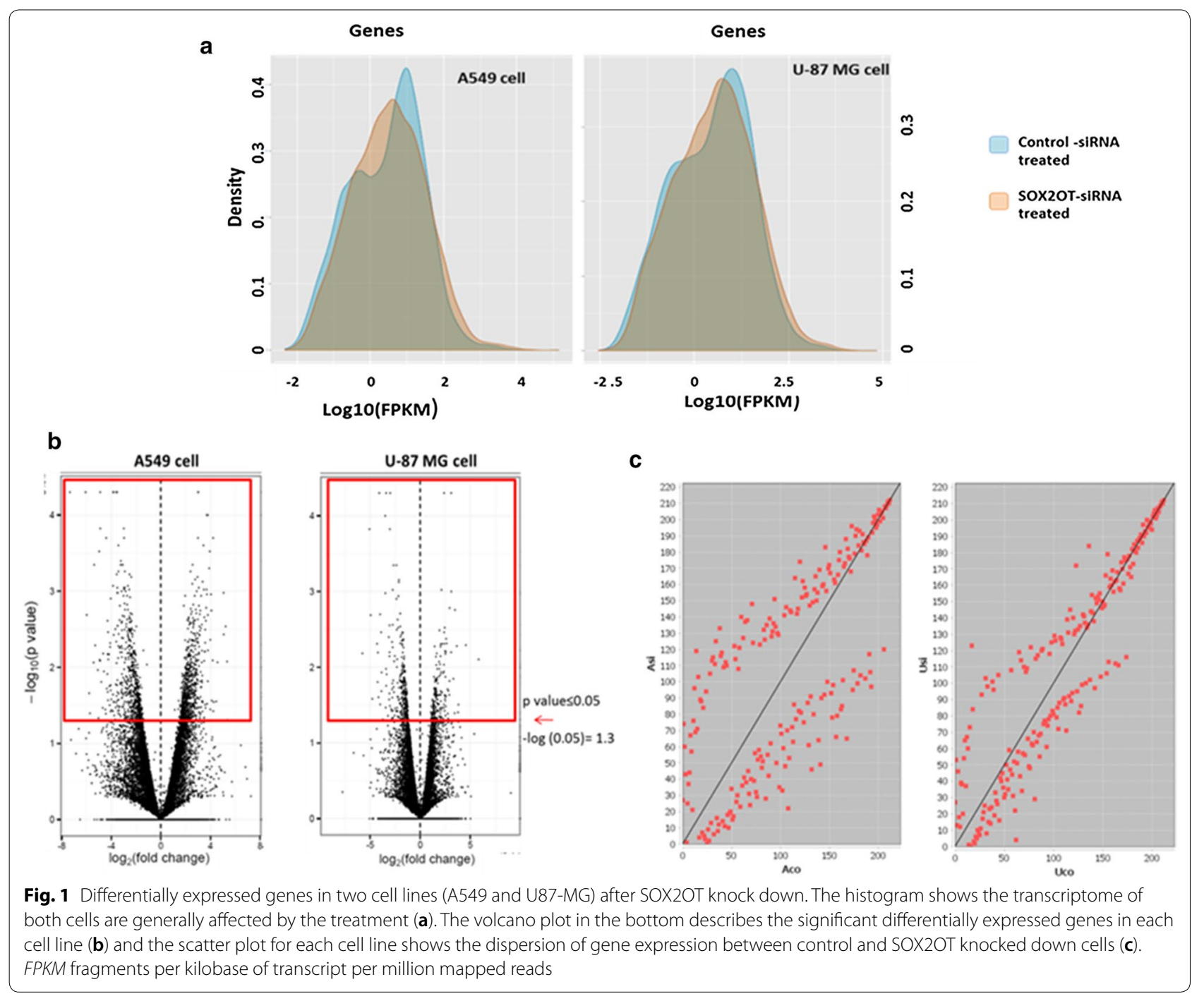

gene list of DEGs in both target cell lines, and we named it: common DEGs. We found 208 genes differentially expressed in both SOX2OT knocked down cell lines. The Venn diagram represented that 90 genes are down regulated and 118 genes are up regulated upon SOX2OT inhibition in Lung cancer cell similar to glioblastoma cell (Fig. 2a). The gene expression value of each sample was used for heat map visualization using geWorkbench platform [29] (Additional file 1: Figure S4). The top differentially expressed genes ordered according the mean of fold change in both cells are shown in Fig. 2b. The GO enrichment of the common DEGs was done with both Bingo and GeneCodis [30] with default parameters and it is available in Additional file 1: Table S2.

The resulted common DEGs list was used for further enrichment and biological process annotation. The most relevant gene ontology terms to Nucleotide binding or DNA replication and cell cycle was summarized in Table 3.

The most related pathway to SOX2OT inhibition was renal cell carcinoma (Kegg: 05211, corrected p value $=0.004953$ ) with 5 genes differentially expressed in RNA sequences of both cells (hif1a, hras, pik3ca, rap1b, $v h l$ ). However, the cell cycle pathway (Kegg: 04110, corrected $\mathrm{p}$ value $=0.03$ ) including the proliferation associated genes ( $r b l 2$, orc4, $c d k 2$, mad1ll1), acquired less score.

The gene network of common DEGs was constructed according the method described in "RNA-sequencing" section. The network was then clustered with the GLayalgorithm [31] to find the most important modules in the network. For more confidence, the edges with weight lower than 0.4 were removed from the network resulting to filtered network with 122 nodes. The clustering resulted to the number of eight modules with the most relevant genes correlated, and the two modules with 
Table 2 Gene ontology term enrichment of differentially expressed genes in the A549 and U87-MG SOX2OT knocked down cells

\begin{tabular}{|c|c|c|c|c|c|c|c|c|c|}
\hline \multicolumn{5}{|c|}{ A549 cell line } & \multicolumn{5}{|c|}{ U87-MG cell line } \\
\hline GO-ID & p-value & Corr p-value & $x$ & Description & GO-ID & p-value & Corr p-value & $\mathrm{x}$ & Description \\
\hline 9987 & $1.83 \mathrm{E}-05$ & $9.26 \mathrm{E}-03$ & 61 & Cellular process & 32502 & $4.68 \mathrm{e}-02$ & $3.40 \mathrm{e}-01$ & 96 & Developmental process \\
\hline 44085 & $1.03 \mathrm{E}-04$ & $1.06 \mathrm{E}-02$ & 21 & Cellular component biogenesis & 6807 & $2.55 e-03$ & $3.19 e-01$ & 76 & $\begin{array}{l}\text { Nitrogen compound metabolic } \\
\text { process }\end{array}$ \\
\hline 7049 & $6.16 \mathrm{E}-05$ & $9.26 \mathrm{E}-03$ & 17 & Cell cycle & 6950 & $2.55 \mathrm{e}-02$ & $3.19 \mathrm{e}-01$ & 58 & Response to stress \\
\hline 22402 & $4.46 \mathrm{E}-04$ & $1.90 \mathrm{E}-02$ & 14 & Cell cycle process & 30154 & $4.98 e-02$ & $3.40 e-01$ & 53 & Cell differentiation \\
\hline 48519 & $8.18 \mathrm{E}-05$ & $9.85 \mathrm{E}-03$ & 13 & $\begin{array}{l}\text { Negative regulation of biological } \\
\text { process }\end{array}$ & 42127 & $5.77 e-03$ & $3.19 e-01$ & 34 & Regulation of cell proliferation \\
\hline 43933 & $2.12 \mathrm{E}-03$ & $4.64 \mathrm{E}-02$ & 13 & $\begin{array}{l}\text { Macromolecular complex subunit } \\
\text { organization }\end{array}$ & 6629 & $3.47 e-02$ & $3.40 \mathrm{e}-01$ & 30 & Lipid metabolic process \\
\hline 278 & $1.47 \mathrm{E}-04$ & $1.18 \mathrm{E}-02$ & 11 & Mitotic cell cycle & 6811 & $4.39 e-02$ & $3.40 \mathrm{e}-01$ & 27 & Ion transport \\
\hline 51301 & $5.24 \mathrm{E}-04$ & $1.95 \mathrm{E}-02$ & 11 & Cell division & 22008 & $2.10 e-02$ & $3.19 e-01$ & 25 & Neurogenesis \\
\hline 22403 & $2.29 \mathrm{E}-03$ & 4.73E-02 & 11 & Cell cycle phase & 48699 & $1.72 \mathrm{e}-02$ & $3.19 \mathrm{e}-01$ & 24 & Generation of neurons \\
\hline 10605 & $3.55 E-04$ & 1.74E-02 & 10 & $\begin{array}{l}\text { Negative regulation of macromol- } \\
\text { ecule metabolic process }\end{array}$ & 8285 & $8.58 \mathrm{e}-03$ & $3.19 e-01$ & 18 & $\begin{array}{l}\text { Negative regulation of cell } \\
\text { proliferation }\end{array}$ \\
\hline 45892 & $6.13 E-05$ & $9.26 \mathrm{E}-03$ & 9 & $\begin{array}{l}\text { Negative regulation of transcrip- } \\
\text { tion, DNA-dependent }\end{array}$ & 55086 & $2.43 \mathrm{E}-02$ & $3.19 \mathrm{E}-01$ & 14 & $\begin{array}{l}\text { Nucleobase, nucleoside and } \\
\text { nucleotide metabolic process }\end{array}$ \\
\hline 45934 & $3.61 \mathrm{E}-04$ & $1.74 \mathrm{E}-02$ & 9 & $\begin{array}{l}\text { Negative regulation of nucleic } \\
\text { acid metabolic process }\end{array}$ & 6954 & $3.08 e-02$ & $3.40 e-01$ & 14 & Inflammatory response \\
\hline 51172 & $3.61 \mathrm{E}-04$ & $1.74 \mathrm{E}-02$ & 9 & $\begin{array}{l}\text { Negative regulation of nitrogen } \\
\text { compound metabolic process }\end{array}$ & 7420 & $4.63 e-02$ & $3.40 e-01$ & 13 & Brain development \\
\hline 6260 & 5.07E-04 & $1.95 \mathrm{E}-02$ & 7 & DNA replication & 6916 & $3.19 \mathrm{E}-02$ & $3.40 \mathrm{E}-01$ & 10 & Anti-apoptosis \\
\hline 9628 & $1.19 \mathrm{E}-03$ & $3.18 \mathrm{E}-02$ & 7 & Response to abiotic stimulus & 6260 & $4.82 \mathrm{e}-02$ & $3.40 e-01$ & 9 & DNA replication \\
\hline 6261 & $2.03 E-03$ & $4.59 E-02$ & 5 & $\begin{array}{l}\text { DNA-dependent DNA replica- } \\
\text { tion }\end{array}$ & 1525 & $4.03 E-02$ & $3.40 E-01$ & 8 & Angiogenesis \\
\hline 10688 & $2.93 \mathrm{E}-04$ & $1.74 \mathrm{E}-02$ & 2 & $\begin{array}{l}\text { Negative regulation of ribosomal } \\
\text { protein gene transcription }\end{array}$ & 51325 & $1.84 \mathrm{e}-02$ & $3.19 e-01$ & 7 & Interphase \\
\hline 30837 & $2.00 E-03$ & 4.59E-02 & 2 & $\begin{array}{l}\text { Negative regulation of actin } \\
\text { filament polymerization }\end{array}$ & 7265 & $2.41 \mathrm{e}-02$ & $3.19 e-01$ & 7 & Ras protein signal transduction \\
\hline 32272 & $2.00 \mathrm{E}-03$ & $4.59 \mathrm{E}-02$ & 2 & $\begin{array}{l}\text { Negative regulation of protein } \\
\text { polymerization }\end{array}$ & 82 & $4.27 e-02$ & $3.40 \mathrm{e}-01$ & 4 & $\begin{array}{l}\text { G1/s transition of mitotic cell } \\
\text { cycle }\end{array}$ \\
\hline 8154 & $2.00 E-03$ & $4.59 E-02$ & 2 & $\begin{array}{l}\text { Actin polymerization or depo- } \\
\text { lymerization }\end{array}$ & 48048 & $8.49 e-03$ & $3.19 e-01$ & 3 & Embryonic eye morphogenesis \\
\hline
\end{tabular}

$\mathrm{X}$ : the number of genes in enriched GO term, corr $\mathrm{p}$-value: FDR corrected $\mathrm{p}$-value

less than 3 nodes were ignored. Then each module was annotated for functional enrichment and the resulted GO terms were used to describe each module (Fig. 3a). Interestingly, we found four gene clusters (modules: 1, 2, 3 and 6) associated with cell cycle or DNA replication and two related with brain or eye development (modules: 5 and 7). The network was then analyzed to find the most important genes, according to the weighted betweenness centrality measurement [32]. Then the 10 top sorted genes with the highest betweenness were extracted in Table 4. According to the modules 1, 2 and 6 (with cell cycle related genes $\mathrm{p}$ value $<2 \times \mathrm{E}-2$ ) and the top 10 hub genes in the network, we selected the number of six genes (bolded in Table 3) for qRT-PCR validation.

\section{Cell cycle associated genes are de-regulated in both cell} lines

Previously, we reported thatSOX2OT knockdown can limit A549 lung cancer cell proliferation with minimal cell cycle perturbation. Here we first measured the effect of SOX2OT inhibition in U-87 MG glioblastoma cancer cell apoptosis and cell cycle progression. Same as our previous report in A549 [21], we observed no apoptosis in SOX2OT knocked down U-87 MG cell line (Additional file 1: Figure $5 \mathrm{~A}$ ), however the population of $\mathrm{G} 2 / \mathrm{M}$ cells were increased approximately $5 \%$ in SOX2OT siRNA treated cells (Additional file 1: Figure 5B). According to the previous reports of SOX2OT function in cell cycle regulation $[17,21]$, we decided to focus on the DEGs related to cell cycle regulation. The qRT-PCR results confirmed the down-regulation of the mitotic cell cycle 
a

\section{Common Down regulated genes}

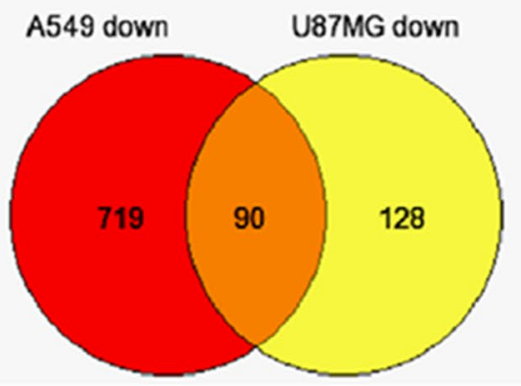

Common up regulated genes
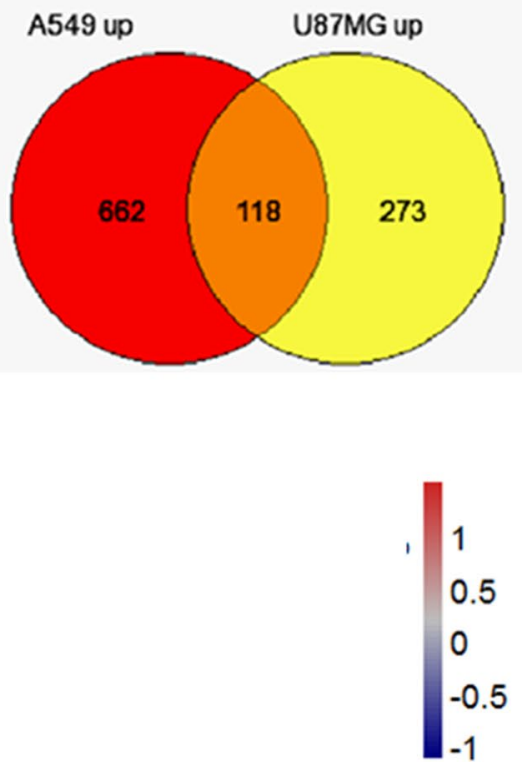

b
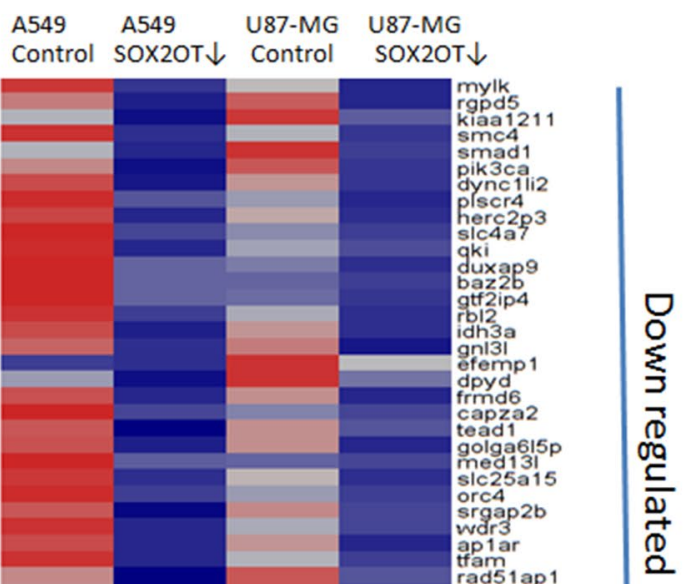

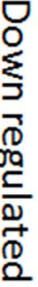

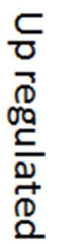

Fig. 2 Common differentially expressed genes in both cell lines. The venn diagram presentation of DEGs was used to find out the gene list differentially expressed in both Lung adenocarcinoma, A549 and glioblastoma, U-87 MG cell lines (http://genevenn.sourceforge.net) (a). The heat map presentation of top common DEGs in siRNA treated or control cell line, which red: high expression value, blue: low expression value and white: mean level of expression value (b)

regulators such as $C D K 2$ and SMC4 in both lung and glioblastoma SOX2OT knocked down cancer cell lines, while the $C D K 2 A P 2$ which is also known to interact and inhibit $C D K 2$ [33], is up-regulated more than two times in treated cells (Fig. 3b). The gene expression changes of other targeted genes (inner centromere protein, guanine nucleotide binding protein-like 3 and actin-related protein 3) were not significant; however we observed 
Table 3 The cell cycle related gene ontology enriched genes in the common DEGs

\begin{tabular}{lll}
\hline GO term & p value & Enriched genes \\
\hline Cell cycle (KEGG) & 0.004 & RBL2, ORC4, CDK2, MAD1L1 \\
Nucleotide binding (MF) & 0.0001 & R3HCC1, HRAS, CARS2, NXF1, ORC4, MVD, G3BP2, PIK3CA, HIPK1, HSPA1B, \\
& & RHOT1, ACTR3, RAP1B, DDX21, GNL3L, CDK2, MYLK, MRPL23, HSPA4L, \\
& CDC34, CHUK, PMVK, PRKD2, SMC4, HSPA1A, DYNC1LI2 \\
G2/M transition of mitotic cell cycle (BP) & $3.23 E-06$ & TAF2, CDK2, NEDD1 \\
Mitotic cell cycle checkpoint (BP) & 0.0018 & ORC4, CDK2, MAD1L1 \\
G1/S transition of mitotic cell cycle (BP) & 0.008 & ORC4, CDK2, CDC34, EIF4E \\
Mitotic cell cycle (BP) & 0.026 & ORC4, CDK2, MAD1L1, INCENP, NEDD1 \\
\hline
\end{tabular}

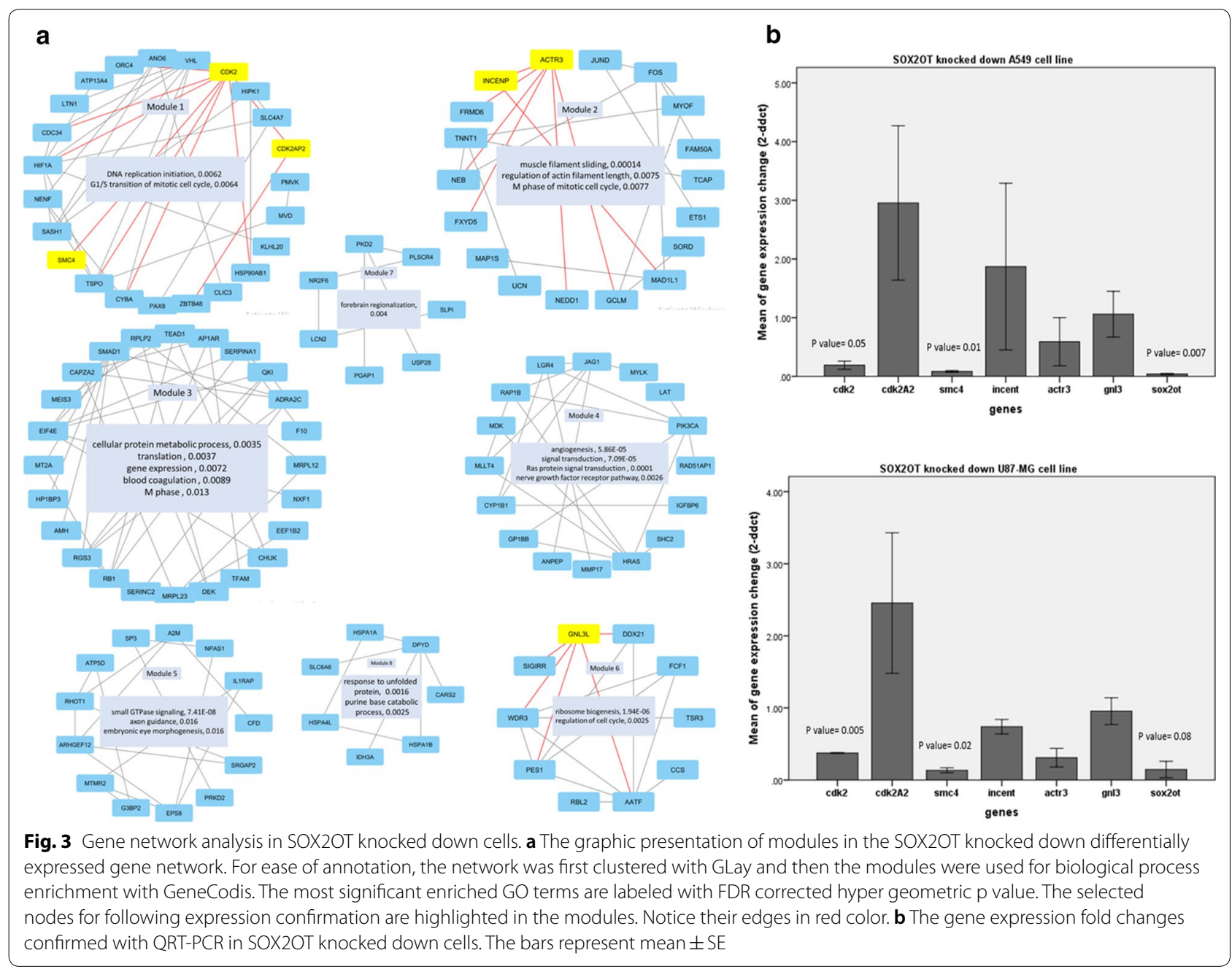

the increased GNL3L expression and decrease ACTR3 expression in both SOX2OT knocked down cancer cell lines same as RNA sequencing results. The GNL3L, which promotes the mitotic Telomeric repeat binding factor 1 [34], and ACTR3is constituent of the ARP2/3 complex of actin nucleator [35] are both important in mitotic progression.

\section{Discussion}

Recently another cancer associated lncRNA, SOX2OT, has been discovered as an embryogenesis and central nervous system regulator [14, 36]. SOX2OT is over expressed in human cancer tissues of lung, esophagus and breast when comparing to normal and it's over expression in tumors is associated with $S O X 2$, which is 
Table 4 The 10 hub genes with highest betweenness

\begin{tabular}{|c|c|c|c|c|}
\hline No. & Symbol & Name & Betweenness & Degree \\
\hline 1 & CDK2 & Cyclin-dependent kinase 2 & 2892.27 & 19 \\
\hline 2 & TSPO & Translocator protein & 1921.052 & 16 \\
\hline 3 & EIF4E & Eukaryotic translation initiation factor 4E & 1787.614 & 12 \\
\hline 4 & SERPINA1 & Serpin peptidase inhibitor, clade A & 1535.751 & 12 \\
\hline 5 & HSPA1A & Heat shock protein family a (hsp70) member $1 \mathrm{a}$ & 1353.741 & 6 \\
\hline 6 & SMAD1 & Smad family member 1 & 1157.476 & 12 \\
\hline 7 & ACTR3 & ARP3 actin-related protein 3 homolog & 1072.58 & 10 \\
\hline 8 & HIF1A & Hypoxia-inducible factor 1-alpha & 980.6956 & 12 \\
\hline 9 & HRAS & Harvey rat sarcoma viral oncogene homolog & 965.7061 & 12 \\
\hline 10 & GNL3L & G Protein Nucleolar 3 Like & 945.4339 & 8 \\
\hline
\end{tabular}

located within SOX2OT [16-18]. We found that according to the KEGG enrichment of the common DEGs deregulated upon SOX2OT inhibition; the pathways related to cancer (Kegg: 05200) is one of the most significant enriched pathway ( $\mathrm{p}$-value $=0.009285)$ with 6 genes (HIF1A, HRAS, CHUK, PIK3CA, CDK2, VHL). This finding highlights $S O X 2 O T$ potential function in cancer development and may help to explain how SOX2OT upregulation can affect tumorigenesis.

It has been reported that the expression of both SOX2 and its overlapping transcript are dynamically co regulated in embryogenesis [14] and decline during stem cell differentiation [18]. Exogenous expression of SOX2OT increase SOX2 more than 20 fold [16]. Furthermore, SOX2OT knockdown is concordant with SOX2 decline in cancer cells $[17,18,21]$. The aforementioned evidences describe why it has been proposed that SOX2OT regulates SOX2; however there is no evidence supporting the direct interaction of these two transcripts.

Notably, it has been reported that 3q27 genetic mutation (SOX2 and SOX2OT containing region) is associated with congenital CNS and eye development disorders [36]; and the normal human brain and lens tissues have been recorded to posse the highest SOX2OT expression level. In vertebrate embryos, SOX2OT expression is dynamically regulated highly expressed in developing central nervous system [14]. Concordantly we found some central nervous system development and maintenance key regulators including: JAG1 [37], MDK [38], PKD2 [39], HES4 [40] and the eye formation regulator such as HIPK1 [41], de-regulated along with SOX2OT inhibition. These findings may suggest a mechanism for SOX2OT in CNS and eye development.

It has been reported that SOX2OT knockdown leads to a G2/M arrest and proliferation inhibition in HCC827 and SKMES-1 lung cancer cell lines with a $E Z H 2$ poly comb protein dependent cyclinB1 and cdc2 regulation mechanism [17]. In our previous study, we found similar SOX2OT knockdown derived anti-proliferative and cell cycle effect in and A549 cells [21] and here in U-87 MG cells. Our results illustrated that gene networks of cell cycle progression or cell proliferation (including: $C D K 2$ and SMC4) are affected in both SOX2OT knock downed cancer cells. However, we did not observed cyclinB1 or EZH2 among SOX2OT associated common DEGs that might be related to low precision of the high throughput RNA-sequencing method. Indeed, we observed other cyclin dependent cell cycle regulators including: cdc6, cdc27, ccnt2, ccnt 1 and ccng2 downregulated and cdc37 upregulated only in A549 cell line transcriptome but not in U87 MG (which is not shown). The functional enrichment of transcription factors or gene regulators revealed that, SOX2OT knock down can potentially change cellular expression of transcription factors which is listed in Additional file 1: Table 3. One of the key cell cycle regulator transcription factors, rbl2 is included among the SOX2OT associated transcription factors; however, it needs more investigation to find out their interaction.

The predicted schematic presentation of the interaction between cell cycle associated common DEGs is summarized in Fig. 4. The interaction between candidate transcription factors and the confirmed DEGs suggest that RB1 and RBL2 are two predicted main transcription factors associated with $S M C 4, C D K 2$ and INCENP, GNL3L (indirectly) expression regulation in SOX2OT knocked down cells.

It has been reported that SOX2OT over-expression in breast cancer cell line can restore the G2/M arrested paclitaxel treated cancer cells [16]. SOX2OT is also associated with the hepatic cancer cell migration and metastasis [42]. Our findings suggest some actin cytoskeleton organization (EPS8, INF2, CAPZA2, TCAP, PLEK2, HRAS, ACTR3) or tubule counterparts 


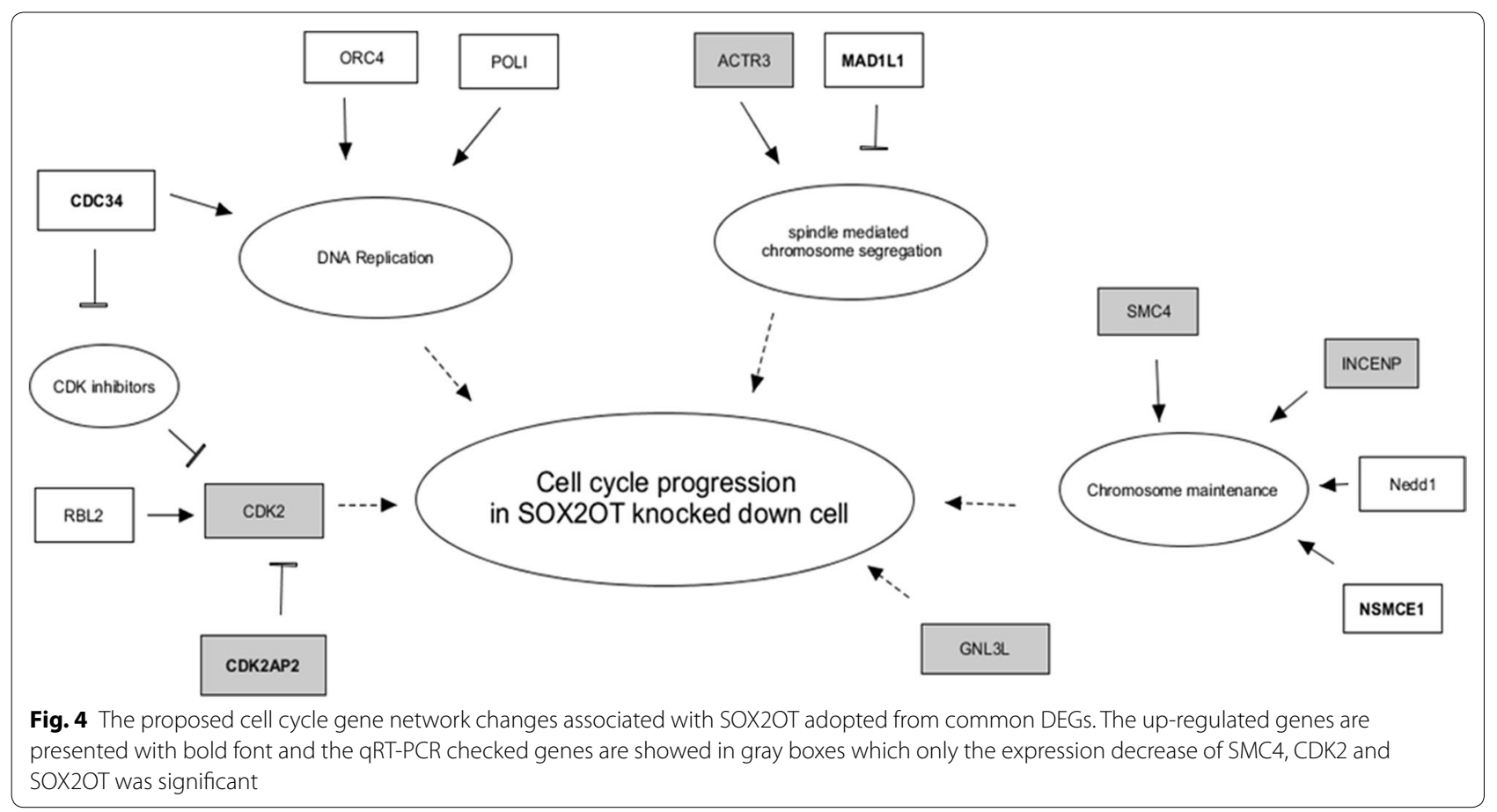

(MAP1S, MTMR2) which provides more evidence to support the probable SOX2OT function in cytoskeleton regulation.

Indeed SOX2OT is reported to bind to PIN2/TERF1interacting telomerase inhibitor 1 (PINX1), in vitro [43]; which can stabilize Telomeric repeat binding factor 1 (TRF1) [44]. GNL3L that is down regulated in SOX2OT inhibited cell can also bind and stabilize TRF1 protein mediatingits mitotic increase and mitosis progression [45]. Also we did not found TRF1 or PINX1 in DEGs since our study was performed at transcription level, then for more completed SOX2OT functional analysis; proteomics or cellular component structure investigation is suggested.

\section{Conclusion}

Altogether, our results illustrate that SOX2OT knock down can significantly alter the gene expression profile of cancer cell lines targeting the cell cycle, proliferation and cytoskeleton related genes. This novel potential function of SOX2OT promises new insights in cancer therapies; however more investigation is necessary to clear the underlying mechanism of SOX2OT relevance to mitotic cell cycle regulation or embryonic development.

\section{Additional file}

Additional file 1: Figure S1. The most associated cancers with the SOX2OT long non-coding RNA. The GENEVESTIGATOR tool was used to find out the most related conditions reported in databases to be associated with SOX2OT expression changes. The top significant conditions are listed in left including two types of brain and lung cancers (A). The schematic presentation of the SOX2OT expression level in different tissues adopted from the MiTranscriptome (B). Figure S2. The most similar conditions to the DEGs in SOX2OT knocked down U87-MG cell line (log2 expression). Figure S3. The most similar conditions to the DEGs in SOX2OT knocked down A549 cell line (log2 expression). Figure S4. The heat map presentation of all the significant common DEGs in siRNA treated or control cell line, which red: high expression value, blue: low expression value and white: mean level of expression value. Figure S5. SOX2OT inhibition effect in apoptosis and cell cycle of cancer cell lines. (A) The apoptotic response of the SOX2OTknocked down U-87 MG was compared with control cells with annexin V/PI staining. As is shown, no annexinV-positive cells (FL1) were detected. (B) The flow cytometry evaluation of Pl-stained (FL3) cell cycle progression in U-87 MG cell is illustrated in tables for control and SOX2OT knocked down cells. Table S1. The complete common DEGs (P value $\leq 0.05$ ) in both cancer cell lines (A549 and U-87MG). Table S2. Functional gene enrichment results of the common DEGS carried out by Bingo or GeneCodis. Table S3. Functional enrichment of transcription associated genes.

\section{Authors' contributions}

MSJ carried out the cell experiments, gene expression assays and prepared the manuscript. MK and BA contributed in bioinformatics analysis of data. SJM and NMS contributed in project design. All authors read and approved the final manuscript. 


\begin{abstract}
Author details
${ }^{1}$ Metabolic Disorders Research Center, Golestan University of Medical Sciences, Gorgan, Iran. ${ }^{2}$ Stem Cell Research Center, Golestan University of Medical Sciences, Po Box: 4934174611 , Gorgan, Iran. ${ }^{3}$ Department of Molecular Genetics, Faculty of Biological Sciences, Tarbiat Modares University, Tehran, Iran. ${ }^{4} \mathrm{OMICS}^{\mathrm{TM}}$ Research Group (Media Teb Gene), Tehran, Iran. ${ }^{5}$ Laboratory of System Biology and Bioinformatics (LBB), University of Tehran, Institute of Biochemistry and Biophysics, Tehran, Iran.
\end{abstract}

\section{Acknowledgements}

Not applicable.

\section{Competing interests}

The authors declare that they have no competing interests.

\section{Availability of data and materials}

The RNAseq data used for this study is available from the corresponding author on reasonable request.

\section{Consent for publication}

Not applicable.

\section{Ethics approval and consent to participate}

Not applicable.

\section{Funding}

This study was supported by Golestan University of Medical Sciences (Grant Number: 9504015082).

\section{Publisher's Note}

Springer Nature remains neutral with regard to jurisdictional claims in published maps and institutional affiliations.

Received: 20 January 2018 Accepted: 14 August 2018

Published online: 05 September 2018

\section{References}

1. Kaikkonen MU, Lam MT, Glass CK. Non-coding RNAs as regulators of gene expression and epigenetics. Cardiovasc Res. 2011;90(3):430-40.

2. Khalil AM, et al. Many human large intergenic noncoding RNAs associate with chromatin-modifying complexes and affect gene expression. Proc Natl Acad Sci. 2009;106(28):11667-72.

3. Rinn JL, Chang HY. Genome regulation by long noncoding RNAs. Ann Rev Biochem. 2012:81:145-66.

4. Katayama S, et al. Antisense transcription in the mammalian transcriptome. Science. 2005;309(5740):1564-6.

5. Wang $X$, et al. The long arm of long noncoding RNAs: roles as sensors regulating gene transcriptional programs. Cold Spring Harb Perspect Biol. 2011;3(1):a003756.

6. Barsyte-Lovejoy D, et al. The c-Myc oncogene directly induces the H19 noncoding RNA by allele-specific binding to potentiate tumorigenesis. Cancer Res. 2006;66(10):5330-7.

7. Matouk IJ, et al. The H19 non-coding RNA is essential for human tumor growth. PLoS ONE. 2007;2(9):e845.

8. Gupta RA, et al. Long non-coding RNA HOTAIR reprograms chromatin state to promote cancer metastasis. Nature. 2010;464(7291):1071-6.

9. Gutschner T, et al. The non-coding RNA MALAT1 is a critical regulator of the metastasis phenotype of lung cancer cells. Can Res. 2013;73(3):1180-9.

10. Liu X-H, et al. The long non-coding RNA HOTAIR indicates a poor prognosis and promotes metastasis in non-small cell lung cancer. BMC Cancer. 2013:13(1):464.

11. Esteller M. Non-coding RNAs in human disease. Nat Rev Genet. 2011;12(12):861-74

12. Taft RJ, et al. Non-coding RNAs: regulators of disease. J Pathol. 2010:220(2):126-39

13. Wapinski O, Chang HY. Long noncoding RNAs and human disease. Trends Cell Biol. 2011;21(6):354-61.
14. Amaral PP, et al. Complex architecture and regulated expression of the Sox2ot locus during vertebrate development. RNA. 2009;15(11):2013-27.

15. Fantes J, et al. Mutations in SOX2 cause anophthalmia. Nat Genet. 2003;33(4):462.

16. Askarian-Amiri ME, et al. Emerging role of long non-coding RNA SOX2OT in SOX2 regulation in breast cancer. PLoS ONE. 2014;9(7):e102140.

17. Hou Z, et al. A long noncoding RNA Sox2ot regulates lung cancer cell proliferation and is a prognostic indicator of poor survival. Int J Biochem Cell Biol. 2014:53:380-8.

18. Shahryari A, et al. Two novel splice variants of SOX2OT, SOX2OT-S1, and SOX2OT-S2 are coupregulated with SOX2 and OCT4 in esophageal squamous cell carcinoma. Stem Cells. 2014;32(1):126-34.

19. Hruz T, et al. Genevestigator v3: a reference expression database for the meta-analysis of transcriptomes. Adv Bioinform. 2008;2008:420747.

20. Iyer MK, et al. The landscape of long noncoding RNAs in the human transcriptome. Nat Genet. 2015;47(3):199.

21. Saghaeian Jazi M, et al. Overexpression of the non-coding SOX2OT variants 4 and 7 in lung tumors suggests an oncogenic role in lung cancer. Tumour Biol. 2016;37(8):10329-38.

22. Langmead B, Salzberg SL. Fast gapped-read alignment with Bowtie 2. Nat Methods. 2012;9(4):357-9.

23. Trapnell C, Roberts A, Goff L, Pertea G, Kim D, Kelley DR, Pimentel H, Salzberg SL, Rinn JL, Pachter L. Differential gene and transcript expression analysis of RNA-seq experiments with TopHat and Cufflinks. Nat Protoc. 2012;7(3):562.

24. Maere S, Heymans K, Kuiper M. BiNGO: a cytoscape plugin to assess overrepresentation of gene ontology categories in biological networks. Bioinformatics. 2005;21(16):3448-9.

25. Szklarczyk D, et al. STRING v10: protein-protein interaction networks, integrated over the tree of life. Nucleic Acids Res. 2015;43(Database issue):D447-52.

26. Warde-Farley D, et al. The GeneMANIA prediction server: biological network integration for gene prioritization and predicting gene function. Nucleic Acids Res. 2010;38(Web Server issue):W214-20.

27. Resnik P. Semantic similarity in a taxonomy: an information-based measure and its application to problems of ambiguity in natural language. J Artif Intell Res. 1999;11:95-130.

28. Guzzi PH, et al. Semantic similarity analysis of protein data: assessment with biological features and issues. Brief Bioinform. 2012;13(5):569-85.

29. Floratos $A$, et al. geWorkbench: an open source platform for integrative genomics. Bioinformatics. 2010;26(14):1779-80.

30. Tabas-Madrid D, Nogales-Cadenas R, Pascual-Montano A. GeneCodis3: a non-redundant and modular enrichment analysis tool for functional genomics. Nucleic Acids Res. 2012;40(Web Server issue):W478-83.

31. Su G, et al. GLay: community structure analysis of biological networks. Bioinformatics. 2010;26(24):3135-7.

32. Tang Y, et al. CytoNCA: a cytoscape plugin for centrality analysis and evaluation of protein interaction networks. Biosystems. 2015;127:67-72.

33. Liu $Q$, et al. Overexpression of DOC-1R inhibits cell cycle G1/S transition by repressing CDK2 expression and activation. Int J Biol Sci. 2013:9(6):541-9.

34. Zhu Q, et al. GNL3L stabilizes the TRF1 complex and promotes mitotic transition. J Cell Biol. 2009:185(5):827-39.

35. Goley ED, Welch MD. The ARP2/3 complex: an actin nucleator comes of age. Nat Rev Mol Cell Biol. 2006;7(10):713-26.

36. Fantes J, et al. Mutations in SOX2 cause anophthalmia. Nat Genet. 2003;33(4):461-3.

37. Irvin DK, et al. Patterns of Jagged1, Jagged2, delta-like 1 and delta-like 3 expression during late embryonic and postnatal brain development suggest multiple functional roles in progenitors and differentiated cells. J Neurosci Res. 2004;75(3):330-43.

38. Winkler C, Yao S. The midkine family of growth factors: diverse roles in nervous system formation and maintenance. Br J Pharmacol. 2014;171(4):905-12.

39. Ohata S, et al. Mechanosensory genes Pkd1 and Pkd2 contribute to the planar polarization of brain ventricular epithelium. J Neurosci. 2015;35(31):11153-68.

40. El Yakoubi W, et al. Hes4 controls proliferative properties of neural stem cells during retinal ontogenesis. Stem Cells. 2012;30(12):2784-95. 
41. Inoue T, et al. Involvement of the Hipk family in regulation of eyeball size, lens formation and retinal morphogenesis. FEBS Lett. 2010;584(14):3233-8.

42. Shi XM, Teng F. Up-regulation of long non-coding RNA Sox2ot promotes hepatocellular carcinoma cell metastasis and correlates with poor prognosis. Int J Clin Exp Pathol. 2015;8(4):4008-14.

43. Siprashvili Z, et al. Identification of proteins binding coding and non-coding human RNAs using protein microarrays. BMC Genom. 2012;13(1):633.
44. Yoo JE, Park YN, Oh BK. PinX1, a telomere repeat-binding factor 1 (TRF1)-interacting protein, maintains telomere integrity by modulating TRF1 homeostasis, the process in which human telomerase reverse Transcriptase (hTERT) plays dual roles. J Biol Chem. 2014;289(10):6886-98.

45. Zhu Q, et al. GNL3L stabilizes the TRF1 complex and promotes mitotic transition. J Cell Biol. 2009;185(5):827-39.
Ready to submit your research? Choose BMC and benefit from:

- fast, convenient online submission

- thorough peer review by experienced researchers in your field

- rapid publication on acceptance

- support for research data, including large and complex data types

- gold Open Access which fosters wider collaboration and increased citations

- maximum visibility for your research: over $100 \mathrm{M}$ website views per year

At BMC, research is always in progress.

Learn more biomedcentral.com/submissions 